\title{
Anesthetic Management of a Patient with Sick Sinus Syndrome during General Anesthesia for Maxillofacial Surgery
}

\author{
Kenichi Satoh ${ }^{*}$, Atsumi Ishizuka1, Ayako Ohashi', Miho Kumagai ${ }^{2}$, Shigeharu Joh' \\ ${ }^{1}$ Division of Dental Anesthesiology, Department of Reconstructive Oral and Maxillofacial Surgery, School of \\ Dentistry, Iwate Medical University, Morioka, Japan \\ ${ }^{2}$ Division of Special Care Dentistry, Department of Developmental Oral Health Science, School of Dentistry, \\ Iwate Medical University, Morioka, Japan \\ Email: satoken@iwate-med.ac.jp
}

Received 2 March 2015; accepted 13 April 2015; published 15 April 2015

Copyright (C) 2015 by authors and Scientific Research Publishing Inc.

This work is licensed under the Creative Commons Attribution International License (CC BY).

http://creativecommons.org/licenses/by/4.0/

(c) (i) Open Access

\section{Abstract}

Sick sinus syndrome (SSS) is a generalized abnormality of cardiac impulse formation. Patients with SSS occasionally need temporary pacing during general anesthesia. The most common issue arising in the perioperative period is electromagnetic interference with device function. We report a case of a 66-year-old man who required temporary cardiac pacing during maxillary cyst extirpation using electrocautery.

\section{Keywords}

Electrocauty, Oral Maxillofacial Surgery, Sick Sinus Syndrome, Temporary Cardiac Pacing

\section{Introduction}

Sick sinus syndrome (SSS) is a generalized abnormality of cardiac impulse formation. Abnormalities encompassed by this syndrome include sinus bradycardia, sinus arrest or exit block, combinations of sinoatrial and atrioventricular nodal conduction disturbances, and atrial tachyarrhythmias [1] [2]. The treatment for symptomatic bradyarrhythmias in patients with SSS is pacemaker placement [1]-[3]. We describe the management of a patient with SSS who required temporary pacing during general anesthesia for maxillofacial surgery.

Written consent was obtained from the patient to publish this report.

\footnotetext{
${ }^{*}$ Corresponding author.
}

How to cite this paper: Satoh, K., et al. (2015) Anesthetic Management of a Patient with Sick Sinus Syndrome during General Anesthesia for Maxillofacial Surgery. Open Journal of Anesthesiology, 5, 53-56.

http://dx.doi.org/10.4236/ojanes.2015.54011 


\section{Case Report}

A 66-year-old man who weighed $73 \mathrm{~kg}$ was admitted with a postoperative maxillary cyst in the left maxillary sinus. His medical history included hypertension and SSS (Class I), which was given the diagnose by medical physician a few years ago. He had been taking the vasodilator amlodipine for 6 years. The patient's left ventricular function was normal with an ejection fraction of $63 \%$. His preoperative electrocardiogram (ECG) showed sinus bradycardia ( $45 \mathrm{bpm})$, a prolonged $P R$ interval of 0.23 seconds and a high voltage in $\mathrm{V}_{1}(0.72 \mathrm{mV})$ and $\mathrm{V}_{5}$ $(2.87 \mathrm{mV})$ (Figure 1). The oral and maxillofacial surgeon consulted the medical doctor in cardiovascular medicine about perioperative management. The medical doctor determined to need for a temporary pacing during general anesthesia because this patient have no subjective symptom at resting or on exercise and there were atrioventricular dissociation due to bradycardia all day, heart rate (HR) at $30 \mathrm{bpm}$ range and cardiac arrest of last for 3 seconds or more all night in Holter ECG monitoring. Before surgery, the medical doctor inserted a temporary pacemaker (Reocor S) into the right ventricular apex via the femoral vein and set at VVI rate $30 \mathrm{ppm}$, pace $3 \mathrm{~V}$ and sense $2 \mathrm{mV}$.

Before anesthetic induction, the patient's HR was $62 \mathrm{bpm}$ and his BP was 158/92 mmHg. Anesthesia was induced with propofol $(120 \mathrm{mg})$ and rocuronium $(50 \mathrm{mg})$; after endotracheal intubation, anesthesia was maintained with sevoflurane (1\% - 1.2\% at gas analysis), remifentanil $(0.1-0.2 \mathrm{mcg} / \mathrm{ml} / \mathrm{h})$, and fentanyl (total dose of $300 \mu \mathrm{g})$. We usually administer sevoflurane or remifentanil at these concentrations and used remifentanil to be more stable for vital sign for oral and maxillofacial surgery. After intubation, the patient's HR and arterial blood pressure (ABP) gradually decreased; immediately before the operation, his HR was 36 - 40 bpm and his BP was 76/38 mm Hg. The pacemaker became active at this point. Ephedrine (6 mg) was administered intravenously, and the patient's HR and ABP were restored to $51 \mathrm{bpm}$ and 108/57 mm Hg, respectively, within 3 minutes. At the same time, local anesthetic ( $2 \mathrm{~mL}$ of $3 \%$ prilocaine) was infiltrated into the maxillary submucosa.

Before the operation began, an electrosurgical unit (Conmed Excalibur Plus PC, Aspen Surgical Systems, USA) with a bipolar active electrode had been prepared for cutting and coagulation; a ground plate had been placed on the patient's left lower back. After the operation began, the patient's HR gradually decreased and ventricular pacing was occasionally initiated. We monitored the patient's hemodynamics (HR, 36 - 52 bpm; systolic ABP, 76 - 120 mm Hg; diastolic ABP, 38 - 64 mm Hg) over the subsequent 150 minutes. Ephedrine (4 or $6 \mathrm{mg}$ ) was intravenously administered twice more when his HR was below $40 \mathrm{bpm}$ and his systolic ABP was below 80 $\mathrm{mm} \mathrm{Hg}$. After each ephedrine injection, the patient's HR increased to 50 - $54 \mathrm{bpm}$ and his systolic ABP increased to around $100 \mathrm{~mm} \mathrm{Hg}$. The surgery and anesthesia were uneventful, and the pacemaker was withdrawn the day after surgery.

Electrocardiogram shows sinus bradycardia, a prolonged $P R$ interval and a high voltage in $V_{1}$ and $V_{5}$.

\section{Discussion}

Sick sinus syndrome is characterized by sinus node dysfunction with an atrial rate inappropriate for physiologic



Figure 1. Preoperative electrocardiogram. 
requirements because the sinus node is unable to perform its pacemaking function [1] [2] [4]. Sinus node dysfunction can be diagnosed on ECG, and SSS can produce a variety of ECG abnormalities, including atrial bradyarrhythmias, atrial tachyarrhythmias, and alternating bradyarrhythmias and tachyarrythmias [1]. Pacemaker implantation is the treatment of choice for symptomatic bradyarrhythmias in patients with SSS [1] [5]. Symptoms of SSS may be variable, intermittent, and difficult to associate with ECG changes [3]. The most common diagnostic method is Holter monitoring [2] [5]. Our patient's preoperative evaluation, which included Holter monitoring, indicated a need for temporary pacing during general anesthesia for maxillofacial surgery.

It is particularly important to carefully consider the details of anesthetic management using temporary cardiac pacing in SSS patients undergoing maxillofacial surgeries involving electrocautery. This is because the most common issue arising in the perioperative period is electromagnetic interference with device function [6] [7]. There are multiple pieces of electrical medical equipment in an operating room, and it is well known that electric cautery can cause interference with the function of cardiac implantable electronic devices (CIEDs). The proper management of potential sources of electromagnetic interference (EMI) associated with electrocautery includes:1) ensuring that the cautery tool and current return pad are positioned so the current pathway does not pass through or near the CIED pulse generator and leads; 2) avoiding proximity of the cautery's electrical field and the pulse generator or leads; 3) using short, intermittent, and irregular bursts of cautery at the lowest feasible energy levels; and 4) using a bipolar electrocautery system or an ultrasonic (harmonic) scalpel if possible [7]. According to the consensus statement prepared by the American Society of Anesthesiologists [7], the likelihood of adverse EMICIED interactions decreases with increasing distance between the EMI source and the pulse generator, and a critical distance of at least 6 inches is specified.

In our case, an electrosurgical unit with a bipolar active electrode was prepared and placed on the operating bed to the side of the patient's legs; the cautery dispersal plate was placed at the patient's left lower back. The pulse generator could be placed around the patient's right ankle because the temporary pacemaker catheter had been inserted via the femoral vein. Therefore, it was possible to keep the active electrode more than 6 inches from the pacing lead of the pacemaker despite the maxillofacial surgery field. Seo [8] reported the possible occurrence of adverse EMI-CIED interactions in patients undergoing radical left neck dissection and maxillectomy when the electrocautery electrode was placed too close to the pacemaker lead, which was inserted through the right subclavian vein. Stone [6] demonstrated that commonly used anesthetic agents do not affect pacing thresholds, although these sequelae of anesthetic management can (e.g., hyperventilation, significant acid-base and/or electrolyte disturbances, significant volume loads, blood transfusion, myocardial ischemia, and high blood concentrations of local anesthetics).

When severe bradycardia or hypotension occurs, anesthetists usually inject atropine sulfate or an adrenergic agonist intravenously; among adrenergic agonists, the drug of choice is ephedrine hydrochloride [8] [9]. We injected ephedrine hydrochloride three times during general anesthesia when the patient's HR decreased to 36 - 40 bpm and his systolic blood pressure fell below $80 \mathrm{~mm} \mathrm{Hg}$ at the same time. Atropine sulfate was not injected because we wanted to rapidly increase HR and BP at the same time. We prepared to administer atropine sulfate and ephedrine hydrochloride and to continuously infuse isoproterenol or dopamine [8] [9].

\section{Conclusion}

We have described the anesthetic management of a patient with SSS who required temporary pacing during general anesthesia for maxillofacial surgery. Use of a temporary pacemaker preoperatively inserted via the femoral vein enabled an uneventful anesthesia and surgery.

\section{Acknowledgements}

None.

\section{References}

[1] Adan, V. and Crown, L.A. (2003) Diagnosis and Treatment of Sick Sinus Syndrome. American Family Physician, 67, 1725-1732.

[2] Mangrum, J.M. and Dimarco, J.P. (2000) The Evaluation and Management of Bradycardia. The New England Journal of Medicine, 342,703-709. http://dx.doi.org/10.1056/NEJM200003093421006

[3] Bigger, J.T. and Reiffel, J.A. (1979) Sick Sinus Syndrome. Annual Review of Medicine, 30, 91-118. 
http://dx.doi.org/10.1146/annurev.me.30.020179.000515

[4] Rodriguez, R.D. and Schocken, D.D. (1990) Update on Sick Sinus Syndrome, a Cardiac Disorder of Aging. Geriatrics, 45, 26-30.

[5] Keller, K.B. and Lemberg, L. (2006) The Sick Sinus Syndrome. American Journal of Critical Care, 15, $226-229$.

[6] Stone, M.E., Salter, B. and Fischer, A. (2011) Perioperative Management of Patients with Cardiac Implantable Electronic Devices. British Journal of Anaesthesia, 107, 116-126. http://dx.doi.org/10.1093/bja/aer354

[7] American Society of Anesthesiologists (2011) Practice Advisory for the Perioperative Management of Patients with Cardiac Implantable Electronic Devices: Pacemakers and Implantable Cardioverter-Defibrillators: An Updated Report by the American Society of Anesthesiologists Task Force on Perioperative Management of Patients with Cardiac Implantable Electronic Devices. Anesthesiology, 114, 247-261.

[8] Seo, K., Takayama, H., Araya, Y., Miura, K., Tanaka, Y., Kobayashi, Y. and Someya, G. (1996) Electromagnetic Interference of an External Temporary Pacemaker during Maxillofacial and Neck Surgery. Anesthesia Progress, 43, 6466.

[9] Liu, J., Yao, G.E., Zhou, H.D., Jiang, X.J. and Chen, Q. (2013) Prevention of Hemodynamic Instability in Extra-Cranial Carotid Angioplasty and Stenting Using Temporary Transvenous Cardiac Pacemaker. Cell Biochemistry and Biophysics, 65, 275-279. http://dx.doi.org/10.1007/s12013-012-9429-z 